\title{
A Clinical Commentary on the Article "Injectable Acellular Hydrogels for Cardiac Repair"
}

\author{
Robert C. Gorman • Joseph H. Gorman III
}

Received: 24 May 2011 /Accepted: 15 June 2011 / Published online: 13 August 2011

(C) Springer Science+Business Media, LLC 2011

Left ventricular (LV) remodeling caused by a myocardial infarction (MI) is responsible for almost $70 \%$ of the five million cases of heart failure in the USA [1]. Over the past 50 years, the establishment of intensive care units, antiarrhythmic drugs, and reperfusion therapy has improved early survival after acute MI. This success has paradoxically contributed to the increased incidence in heart failure. While the use of optimal pharmacologic therapy can slow the progression to heart failure after MI, the incidence of the disease continues to grow, and survival rates, once clinical symptoms appear, have not increased appreciably since the 1970s [2]. The outlook for patients with end-stage heart failure remains particularly poor.

Dissatisfaction with the treatment options for end-stage heart failure has resulted in an increased interest in developing interventions that can be implemented early after MI with the intent of slowing or arresting the progression to heart failure. Cellular therapy with any number of cell lines has been championed by many investigators as a possible means of regenerating myocardium and ameliorating adverse LV remodeling after MI. In spite of extensive enthusiasm for this therapeutic approach, regeneration of electromechanically integrated myocardium in the infarct zone has never been demonstrated to occur in patients or in large animal infarct models.

Early infarct expansion or stretching has been associated with poor long-term prognosis [3] and has been identified as the mechanical phenomenon that initiates and sustains the process of adverse post-MI LV remodeling that leads to

This article is a commentary on the following article: $10.1007 / \mathrm{s} 12265$ 011-9291-1.

R. C. Gorman $(\bowtie) \cdot$ J. H. Gorman III

Department of Surgery and the Gorman Cardiovascular Research

Group, University of Pennsylvania, Philadelphia,

500 S. Ridgeway Avenue,

Glenolden, PA 19036, USA

e-mail: gormanr@uphs.upenn.edu heart failure $[4,5]$. Infarct expansion results from changes in the material properties (increased compliance) and geometry (thinning) of the infarct region that occur early after MI and progress during adverse remodeling. Infarct expansion causes increased mechanical stress not only in the infarcted zone but also in myocardial regions outside the infarction, especially in the adjacent borderzone region, putting these regions at a mechanical disadvantage $[6,7]$. Increased regional stress is also the impetus for several maladaptive biologic processes, such as myocyte apoptosis and matrix metalloproteinase activation that inherently alter the contractile properties of normally perfused myocardium. Once initiated, these maladaptive biologic processes lead to a heart failure phenotype that is difficult to reverse by medical or surgical means.

Clinical and laboratory experience has taught us that it is useful to conceptualize the post-MI remodeling process as a patholophysiologic spectrum. Very early after coronary occlusion, the infarct zone ceases to contract and becomes subjected to abnormal "mechanical loading" by the contractile force of the remaining perfused segments of the heart which in turn leads to increased mechanical stress in the infarct and perfused myocardium adjacent to and remote from the infarct. With time, this purely mechanical pathology initiates inherent, complex, and irreversible biologic changes in perfused myocardium that are manifest to clinicians as the classic heart failure phenotype.

Based on this understanding of the pathogenesis of infarction-induced heart failure, we identified the prevention of infarct expansion as a potentially important therapeutic goal. Nearly 15 years ago, we hypothesized that adverse post-MI LV remodeling could be ameliorated by preventing infarct expansion with mechanical restraint [8]. In a series of experiments that employed a variety of heart-wrapping techniques, we definitively demonstrated that the prevention of infarct expansion had a powerful salutary effect of LV remodeling [8-14]. 
Translating these proof-of-concept preclinical studies to an efficacious clinical treatment platform will be dependent on developing minimally invasive and effective technologies for limiting infarct expansion in patients identified to be at high risk for adverse LV remodeling early after MI. As the benefit-to-risk ratio increases, such a therapy could be offered to increasing segments of the post-MI population potentially leading to a profound effect on patient longevity and well-being. The implications for the cost of health care in an aging population could also be significant.

Tous and colleges have comprehensively reviewed the emerging therapeutic concept of limiting infarct expansion by the administration of injectable acellular hydrogels [15]. This new treatment paradigm is exciting because it raises the real possibility of limiting adverse LV remodeling by the percutaneous, catheter-based delivery of precisely engineered biomaterials to the infarct in the early post-MI period. Optimized materials would act to stiffen and thicken the infarct resulting in normalized LV wall stress and attenuated LV remodeling. The concept is innovative in that it represents a profound paradigm shift in treatment strategy for heart failure: palliative to preventive, pharmacologic to mechanical, invasive surgery to a minimally invasive percutaneous catheter-based approach.

A range of injectable biomaterials have been studied to achieve this therapeutic goal, including biological materials such as alginate, fibrin, and derivatives of the extracellular matrix, as well as synthetic materials like self-assembling peptides and thermoresponsive gels [15]. The materials tested to date have had a wide range of mechanical and resorptive properties and have most typically been common formulations developed for other applications. Additionally, most of the material systems that have undergone preclinical testing have had fixed material properties and were not amenable to optimization. Thus, there is a need for a material system that can be engineered to allow optimization with regard to stiffness, degradation, and catheter delivery. Finally, the majority of the materials studied so far have only been investigated in rodent models of infarction; effective translation to patients will be dependent on the routine testing of all proposed materials in clinically relevant large animal models of infarction-induced LV remodeling.

Extensive work by material scientists, engineers, and physicians will be required to establish this emerging therapeutic paradigm. The initial goal should be the rational design and development of the optimal infarct-modifying material. Subsequent studies will be needed to develop the delivery technique and material localization methods that will allow for direct clinical translation. Although much work remains to be done, the clinical goal of preventing the progressive spiral to heart failure after MI seems finally to be within our reach.

\section{References}

1. Gheorghiade, M., \& Bonow, R. O. (1998). Chronic heart failure in the United States - a manifestation of coronary artery disease. Circulation, 97, 282-289.

2. Levy, D., Kenchaiah, S., Larson, M. G., Benjamin, E. J., Kupka, M. J., Ho, K. K., et al. (2002). Long-term trends in the incidence of and survival with heart failure. The New England Journal of Medicine, 347, 1397-1402.

3. Weisman, H. F., \& Healy, B. (1987). Myocardial infarct expansion, infarct extension, and reinfarction - pathophysiologic concepts. Progress in Cardiovascular Diseases, 30, 73-110.

4. Jackson, B. M., Gorman, J. H., Moainie, S. L., Guy, T. S., Narula, N., Narula, J., et al. (2002). Extension of borderzone myocardium in postinfarction dilated cardiomyopathy. Journal of the American College of Cardiology, 40, 1160-1167. discussion, 1168-1171.

5. Jackson, B. M., Gorman, J. H., Salgo, I. S., Moainie, S. L., Plappert, T., St John-Sutton, M., et al. (2003). Border zone geometry increases wall stress after myocardial infarction: contrast echocardiographic assessment. American Journal of Physiology. Heart and Circulatory Physiology, 284, H475-H479.

6. Guccione, J. M., Moonly, S. M., Moustakidis, P., Costa, K. D., Moulton, M. J., Ratcliffe, M. B., et al. (2001). Mechanism underlying mechanical dysfunction in the border zone of left ventricular aneurysm: a finite element model study. The Annals of Thoracic Surgery, 71, 654-662.

7. Gorman, R. C., \& Gorman, J. H., III. (2001). Mechanism underlying mechanical dysfunction in the borderzone left ventricular aneurysm. A finite element model study. Invited commentary. The Annals of Thoracic Surgery, 71, 662.

8. Kelley, S. T., Malekan, R., Jackson, B. M., Gorman, J. H., III, Plappert, T., Gorman, R. C., et al. (1999). Restraining infarct expansion preserves left ventricular geometry and function after acute anteroapical infarction. Circulation, 99, 135-142.

9. Moainie, S. L., Guy, T. S., Gorman, J. H., III, Jackson, B. M., Plappert, T., St John Sutton, M., et al. (2002). Infarct restraint attenuates remodeling and reduces chronic ischemic mitral regurgitation after posterolateral infarction. The Annals of Thoracic Surgery, 74, 444-449.

10. Pilla, J. J., Blom, A. S., Brockman, D. J., Bowen, F., Yuan, Q., Giammarco, J., et al. (2002). Ventricular constraint using the acorn cardiac support device reduces myocardial akinetic area in an ovine model of acute infarction. Circulation, 106, I207-I211.

11. Enomoto, Y., Gorman, J. H., III, Moainie, S. L., Jackson, B. M., Parish, L. M., Plappert, T., et al. (2005). Early ventricular restraint after myocardial infarction: extent of the wrap determines the outcome of remodeling. The Annals of Thoracic Surgery, 79, 881887.

12. Blom, A. S., Pilla, J. J., Gorman, R. C., Gorman, J. H., III, Mukherjee, R., Spinale, F. G., et al. (2005). Infarct size reduction and attenuation of global left ventricular remodeling with the CorCap $^{\mathrm{TM}}$ cardiac support device following acute myocardial infarction in sheep. Heart Failure Reviews, 10, 125-139.

13. Pilla, J. J., Blom, A. S., Gorman, J. H., III, Brockman, D. J., Affuso, J., Parish, L. M., et al. (2005). Early post infarction ventricular restraint improves borderzone wall thickening dynamics during remodeling. The Annals of Thoracic Surgery, 80, $2257-$ 2262.

14. Blom, A. S., Mukherjee, R., Pilla, J. J., Lowry, A. S., Yarbrough, W. M., Mingoia, J. T., et al. (2005). A cardiac support device modifies left ventricular geometry and myocardial structure after myocardial infarction. Circulation, 112, 1274-1283.

15. Tous, E., Purcell, B., Ifkovits, J.L., Burdick, J.A. (2011) Injectable acellular hydrogels for cardiac repair. Journal of Cardiovascular Translational Research. (In press). 